\title{
Decompositions of Symmetry Using Odds-Symmetry for Square Contingency Tables
}

\author{
Shuji Ando* and Kyohei Aoba \\ Department of Information and Computer Technology, Faculty of Engineering, Tokyo University of Science, \\ Katsushika-ku, Tokyo, 125-8585, Japan \\ Email: shuji.ando@rs.tus.ac.jp
}

\begin{abstract}
For square contingency tables, many decompositions of the symmetry model were given. If the symmetry model does not hold, the decomposition of symmetry is useful to analyze the cause that the symmetry model fits poorly. The present paper shows the decomposition of the symmetry model using odds-symmetry, and the test statistic for the symmetry model is equal to the sum of those for decomposed models. This paper also gives the decomposition of the conditional symmetry model using odds-symmetry. By comparing the existing decompositions of the symmetry model, we show that the proposed decompositions are useful.
\end{abstract}

Keywords: Marginal symmetry, ordinal category, partitioning, test statistic.

\section{Introduction}

Consider an $R \times R$ square contingency table with the same row and column classifications. Let $p_{i j}$ denote the probability that an observation will fall in the $i$ th row and $j$ th column of the table $(i=1, \ldots, R ; j=$ $1, \ldots, R)$. The symmetry $(\mathrm{S})$ model is defined by

$$
p_{i j}=p_{j i} \quad(i \neq j),
$$

(Bowker, 1948; Bishop, Fienberg and Holland, 1975, p. 282). The S model indicates that the probability that an observation will fall in the $(i, j)$ cell, $i \neq j$, is equal to the probability that the observation falls in the symmetric $(j, i)$ cell.

The marginal homogeneity $(\mathrm{MH})$ model is defined by

$$
p_{i \cdot}=p_{\cdot i} \quad(i=1, \ldots, R),
$$

where $p_{i}=\sum_{t=1}^{R} p_{i t}$ and $p_{\cdot i}=\sum_{s=1}^{R} p_{s i}$ (Stuart, 1955). The MH model indicates that the row marginal distribution is identical to the column marginal distribution.

The quasi-symmetry (QS) model is defined by

$$
p_{i j}=\frac{\alpha_{j}}{\alpha_{i}} p_{j i} \quad(i<j),
$$

(Caussinus, 1965). The QS model with $\left\{\alpha_{i}=\alpha_{j}\right\}$ is identical to the $\mathrm{S}$ model. Denote the odds ratio for rows $i$ and $j(i<j)$, and columns $s$ and $t(s<t)$ by $\theta_{(i<j ; s<t)}$, where $\theta_{(i<j ; s<t)}=\left(p_{i s} p_{j t}\right) /\left(p_{j s} p_{i t}\right)$. The QS model is expressed as

$$
\theta_{(i<j ; s<t)}=\theta_{(s<t ; i<j)} \quad(i<j ; s<t) .
$$

Thus the QS model has characterization in terms of symmetry of odds ratio.

For square contingency tables with ordered categories, McCullagh (1978) considered the conditional symmetry (CS) model, defined by

$$
p_{i j}=\delta p_{j i} \quad(i<j) .
$$

This model with $\delta=1$ is identical to the $\mathrm{S}$ model.

Tomizawa (1985) considered two kinds of odds-symmetry (OS) models as follows:

$$
H_{R O S}: p_{i j} / p_{i, j+1}=p_{j i} / p_{j+1, i} \quad(i<j) ;
$$




$$
H_{C O S}: p_{i-1, j} / p_{i j}=p_{j, i-1} / p_{j i} \quad(i<j) \text {. }
$$

The ROS model indicates that the odds whose column value is $j$ instead of $j+1$ in row $i$ with respect to the upper-right triangle of the table are equal to the symmetric odds whose row value is $j$ instead of $j+1$ in column $i$ with respect to the lower-left triangle of the same table. The COS model also has similar properties. The ROS and COS models can also be expressed as

$$
\begin{array}{ll}
H_{R O S}: p_{i j}=\gamma_{i} p_{j i} & (i<j) ; \\
H_{C O S}: p_{i j}=s_{j} p_{j i} & (i<j) .
\end{array}
$$

The ROS and COS models with $\left\{\gamma_{i}=\delta\right\}$ and $\left\{s_{j}=\delta\right\}$ are identical to the CS model, respectively. Also the ROS and COS models with $\left\{\gamma_{i}=1\right\}$ and $\left\{s_{j}=1\right\}$ are identical to the $\mathrm{S}$ model, respectively.

The global symmetry (GS) model is defined by

$$
\sum_{i<j} \sum_{i j} p_{i>j} \sum_{i>} p_{i j}
$$

(Read, 1977).

For square contingency tables with nominal categories, Caussinus (1965) gave the theorem that the S model holds if and only if both the QS and MH models hold. Also, Tomizawa and Tahata (2007) showed that for large sample, a test statistic for the $\mathrm{S}$ model is approximately equal to the sum of those for the QS and MH models.

For square contingency tables with ordered categories, Read (1977) showed the decomposition of the $\mathrm{S}$ model using the CS and GS models, and the likelihood ratio chi-square test statistic for the $\mathrm{S}$ model is equal to the sum of those for decomposed models. Other many decompositions of the symmetry model were given (see, for example, Tahata and Tomizawa (2014), Kateri, Gottard, and Tarantola (2017)). So, we are interested in considering decompositions of the $\mathrm{S}$ model using OS models. We are also interested in considering decompositions of the CS model using OS models.

The purpose of this paper is (1) to give decompositions of the S model using OS models, (2) to show partitioning for goodness-of-fit test of the S model, and (3) to give decompositions of the CS model using OS models.

\section{Decompositions of Symmetry Using Odds-Symmetry}

In order to show decompositions using OS models, we shall introduce models below.

Tomizawa (1984) considered two kinds of marginal symmetry (MS) models as follows:

$$
\begin{gathered}
H_{R M S}: p_{i \cdot}^{+}=p_{\cdot i}^{-} \quad(i=1, \ldots, R-1) \\
H_{C M S}: p_{\cdot i}^{+}=p_{i .}^{-} \quad(i=2, \ldots, R)
\end{gathered}
$$

where

$$
p_{i .}^{+}=\sum_{k=i+1}^{R} p_{i k}, \quad p_{\cdot i}^{-}=\sum_{k=i+1}^{R} p_{k i}, \quad p_{\cdot i}^{+}=\sum_{k=1}^{i-1} p_{k i} \quad \text { and } \quad p_{i \cdot}^{-}=\sum_{k=1}^{i-1} p_{i k} .
$$

Also, Tomizawa (1984) considered two kinds of marginal asymmetry (MAS) models as follows:

$$
\begin{gathered}
H_{R M A S}: p_{i \cdot}^{+}=\tau_{1} p_{\cdot i}^{-} \quad(i=1, \ldots, R-1) ; \\
H_{C M A S}: p_{\cdot i}^{+}=\tau_{2} p_{i .}^{-} \quad(i=2, \ldots, R) .
\end{gathered}
$$

Let $X$ and $Y$ denote the row and column variables, respectively. Under the RMAS model, $\tau_{1}>1$ is equivalent to $\operatorname{Pr}(X<Y)>\operatorname{Pr}(X>Y)$ and $\tau_{1}<1$ is equivalent to $\operatorname{Pr}(X<Y)<\operatorname{Pr}(X>Y)$. Moreover note that a special case of RMAS obtained by putting $\tau_{1}=1$ is the RMS model. The CMAS model also has similar properties. We obtain decompositions of the $\mathrm{S}$ model using OS models as follows. 
Theorem 1: The $S$ model holds if and only if both the ROS and RMS models hold.

Proof: If the S model holds, then both the ROS and RMS models hold. Assuming that the ROS and RMS models hold, then we shall show that the S model holds. From the both ROS and RMS models hold, we get

$$
\gamma_{i} \sum_{k=i+1}^{R} p_{k i}=\sum_{k=i+1}^{R} p_{k i} \quad(i=1, \ldots, R-1) .
$$

If $\left\{\gamma_{i}>1\right\}$ in the ROS model, we see

$$
\gamma_{i} \sum_{k=i+1}^{R} p_{k i}>\sum_{k=i+1}^{R} p_{k i} \quad(i=1, \ldots, R-1)
$$

If $\left\{\gamma_{i}<1\right\}$ in the ROS model, we see

$$
\gamma_{i} \sum_{k=i+1}^{R} p_{k i}<\sum_{k=i+1}^{R} p_{k i} \quad(i=1, \ldots, R-1) .
$$

Therefore, we obtain $\left\{\gamma_{i}=1\right\}$. Namely, the $\mathrm{S}$ model holds. The proof is completed.

Theorem 2: The $S$ model holds if and only if both the COS and CMS models hold.

The proof of Theorem 2 is omitted because it is obtained in a similar way to the proof of theorem 1. Next, we obtain the following theorems.

Theorem 3: The CS model holds if and only if both the ROS and RMAS models hold.

Theorem 4: The CS model holds if and only if both the COS and CMAS models hold.

Theorem 5: The RMS model holds if and only if both the RMAS and GS models hold.

Theorem 6: The CMS model holds if and only if both the CMAS and GS models hold.

The proofs of Theorems 3 to 6 are omitted because they are obtained in a similar way to the proof of theorem 1. From Theorems 1, 2, 5, 6, we obtain the following corollaries.

Corollary 1: The $S$ model holds if and only if all the ROS, RMAS and GS models hold.

Corollary 2: The $S$ model holds if and only if all the COS, CMAS and GS models hold.

\section{$3 \quad$ Partitioning Test Statistic}

Assume that a multinomial distribution applies to the $R \times R$ table. Let $n_{i j}$ denote the observed frequency in the $i$ th row and $j$ th column of the $R \times R$ table $(i=1, \ldots, R ; j=1, \ldots, R)$, with $n=\sum \sum n_{i j}$.

The maximum likelihood estimates (MLEs) of expected frequencies $\left\{m_{i j}\right\}$ under the S, CS, ROS, COS, RMS, CMS, RMAS, CMAS, GS models are expressed as the closed-forms as follows:

(a) The MLEs of $\left\{m_{i j}\right\}$ under the $\mathrm{S}$ model is

$$
\hat{m}_{i j}=\left\{\begin{array}{lll}
\frac{n_{i j}+n_{j i}}{2} & \text { for } & i \neq j \\
n_{i j} & \text { for } & i=j .
\end{array}\right.
$$


(b) The MLEs of $\left\{m_{i j}\right\}$ under the CS model is

$$
\hat{m}_{i j}=\left\{\begin{array}{lll}
\frac{n_{u}}{n_{u}+n_{l}}\left(n_{i j}+n_{j i}\right) & \text { for } & i<j, \\
\frac{n_{l}}{n_{u}+n_{l}}\left(n_{i j}+n_{j i}\right) & \text { for } & i>j, \\
n_{i j} & \text { for } & i=j,
\end{array}\right.
$$

where

$$
n_{u}=\sum_{i<j} \sum_{i j} n_{i j} \quad \text { nnd } \quad n_{l}=\sum_{i>j} \sum_{i j} n_{i j}
$$

(c) The MLEs of $\left\{m_{i j}\right\}$ under the ROS model is

$$
\hat{m}_{i j}=\left\{\begin{array}{lll}
\frac{n_{i \cdot}^{+}}{n_{i \cdot}^{+}+n_{\cdot}^{-}}\left(n_{i j}+n_{j i}\right) & \text { for } & i<j, \\
\frac{n_{\cdot j}^{-}}{n_{j .}^{+}+n_{\cdot j}^{-}}\left(n_{i j}+n_{j i}\right) & \text { for } & i>j, \\
n_{i j} & \text { for } & i=j,
\end{array}\right.
$$

where

$$
n_{i \cdot}^{+}=\sum_{k=i+1}^{R} n_{i k} \quad \text { and } \quad n_{\cdot i}^{-}=\sum_{k=i+1}^{R} n_{k i} .
$$

(d) The MLEs of $\left\{m_{i j}\right\}$ under the COS model is

$$
\hat{m}_{i j}=\left\{\begin{array}{lll}
\frac{n_{\cdot j}^{+}}{n_{\cdot j}^{+}+n_{j .}^{-}}\left(n_{i j}+n_{j i}\right) & \text { for } & i<j, \\
\frac{n_{i \cdot}^{-}}{n_{\cdot i}^{+}+n_{i .}^{-}}\left(n_{i j}+n_{j i}\right) & \text { for } & i>j, \\
n_{i j} & \text { for } & i=j,
\end{array}\right.
$$

where

$$
n_{\cdot i}^{+}=\sum_{k=1}^{i-1} n_{k i} \quad \text { and } \quad n_{i \cdot}^{-}=\sum_{k=1}^{i-1} n_{i k}
$$

(e) The MLEs of $\left\{m_{i j}\right\}$ under the RMS model is

$$
\hat{m}_{i j}=\left\{\begin{array}{lll}
\frac{n_{i j}\left(n_{i \cdot}^{+}+n_{\cdot \cdot}^{-}\right)}{2 n_{i \cdot}^{+}} \text {for } & i<j, \\
\frac{n_{i j}\left(n_{j \cdot}^{+}+n_{\cdot j}^{-}\right)}{2 n_{\cdot j}^{-}} \text {for } & i>j, \\
n_{i j} & \text { for } & i=j .
\end{array}\right.
$$

(f) The MLEs of $\left\{m_{i j}\right\}$ under the CMS model is

$$
\hat{m}_{i j}=\left\{\begin{array}{lll}
\frac{n_{i j}\left(n_{\cdot j}^{+}+n_{j .}^{-}\right)}{2 n_{\cdot j}^{+}} & \text {for } & i<j, \\
\frac{n_{i j}\left(n_{\cdot \cdot}^{+}+n_{i \cdot}^{-}\right)}{2 n_{i \cdot}^{-}} & \text {for } & i>j, \\
n_{i j} & \text { for } & i=j .
\end{array}\right.
$$


(g) The MLEs of $\left\{m_{i j}\right\}$ under the RMAS model is

$$
\hat{m}_{i j}=\left\{\begin{array}{lll}
\frac{n_{u} n_{i j}\left(n_{i \cdot}^{+}+n_{\cdot}^{-}\right)}{\left(n_{u}+n_{l}\right) n_{i \cdot}^{+}} & \text {for } & i<j, \\
\frac{n_{l} n_{i j}\left(n_{j .}^{+}+n_{\cdot j}^{-}\right)}{\left(n_{u}+n_{l}\right) n_{\cdot j}^{-}} & \text {for } & i>j, \\
n_{i j} & \text { for } & i=j .
\end{array}\right.
$$

(h) The MLEs of $\left\{m_{i j}\right\}$ under the CMAS model is

$$
\hat{m}_{i j}=\left\{\begin{array}{lll}
\frac{n_{u} n_{i j}\left(n_{\cdot j}^{+}+n_{j}^{-}\right)}{\left(n_{u}+n_{l}\right) n_{\cdot j}^{+}} & \text {for } & i<j, \\
\frac{n_{l} n_{i j}\left(n_{\cdot i}^{+}+n_{i \cdot}^{-}\right)}{\left(n_{u}+n_{l}\right) n_{i \cdot}^{-}} & \text {for } & i>j, \\
n_{i j} & \text { for } & i=j .
\end{array}\right.
$$

(i) The MLEs of $\left\{m_{i j}\right\}$ under the GS model is

$$
\hat{m}_{i j}=\left\{\begin{array}{lll}
\frac{\left(n_{u}+n_{l}\right) n_{i j}}{2 n_{u}} & \text { for } & i<j, \\
\frac{\left(n_{u}+n_{l}\right) n_{i j}}{2 n_{l}} & \text { for } & i>j, \\
n_{i j} & \text { for } & i=j .
\end{array}\right.
$$

Each model can be tested for goodness-of-fit by, e.g., the likelihood ratio chi-square statistic (denote by $G^{2}$ ) with the corresponding degrees of freedom (df). The test statistic $G^{2}$ of model $M$ is given by

$$
G^{2}(M)=2 \sum_{i=1}^{R} \sum_{j=1}^{R} n_{i j} \log \left(\frac{n_{i j}}{\hat{m}_{i j}}\right) .
$$

On partitioning test statistic for models in Theorem 1 to 6 , we obtain the following theorems.

Theorem 7: The following equation holds:

$$
G^{2}(S)=G^{2}(R O S)+G^{2}(R M S) .
$$

Proof: We see that $\left(n_{i j} / \hat{m}_{i j}\right)$ under the $\mathrm{S}$ model is equal to the product of $\left(n_{i j} / \hat{m}_{i j}\right)$ under the ROS model and that under the RMS model. Therefore, the proof is completed.

Theorem 8: The following equation holds:

$$
G^{2}(S)=G^{2}(C O S)+G^{2}(C M S) .
$$

Theorem 9: The following equation holds:

$$
G^{2}(C S)=G^{2}(R O S)+G^{2}(R M A S) .
$$

Theorem 10: The following equation holds:

$$
G^{2}(C S)=G^{2}(C O S)+G^{2}(C M A S) .
$$


Theorem 11: The following equation holds:

$$
G^{2}(R M S)=G^{2}(R M A S)+G^{2}(G S) .
$$

Theorem 12: The following equation holds:

$$
G^{2}(C M S)=G^{2}(C M A S)+G^{2}(G S) .
$$

The proofs of Theorems 8 to 12 are omitted because they are obtained in a similar way to the proof of theorem 7. From Theorems 7, 8, 11, 12, we obtain the following corollaries.

Corollary 3: The following equation holds:

$$
G^{2}(S)=G^{2}(R O S)+G^{2}(R M A S)+G^{2}(G S) .
$$

Corollary 4: The following equation holds:

$$
G^{2}(S)=G^{2}(C O S)+G^{2}(C M A S)+G^{2}(G S) .
$$

\section{An Example}

Consider the data in Table 1, taken from Tominaga (1979, p. 132).

Table 1. The data describe the cross-classification of father's and son's occupational status categories in Japan which was examined in 1975 from Tominaga (1979, p. 132). (The parenthesized values are maximum likelihood estimates of expected frequencies under the COS model.)

\begin{tabular}{cccccc}
\hline Father's & \multicolumn{5}{c}{ Son's status } \\
\cline { 2 - 5 } status & $(1)$ & $(2)$ & $(3)$ & $(4)$ & Total \\
\hline$(1)$ & 127 & 101 & 54 & 12 & 294 \\
& $(127.00)$ & $(101.00)$ & $(62.02)$ & $(7.40)$ & \\
$(2)$ & 86 & 207 & 125 & 13 & 431 \\
& $(86.00)$ & $(207.00)$ & $(116.98)$ & $(13.40)$ & \\
$(3)$ & 78 & 124 & 310 & 24 & 536 \\
& $(69.98)$ & $(132.02)$ & $(310.00)$ & $(28.20)$ & \\
$(4)$ & 109 & 206 & 437 & 325 & 1077 \\
& $(113.60)$ & $(205.60)$ & $(432.80)$ & $(325.00)$ & \\
\hline Total & 400 & 638 & 926 & 374 & 2338 \\
\hline Note: Status is (1) Upper non-manual, (2) Non-manual,
\end{tabular}

(3) Manual and (4) Agriculture.

Table 2 gives the values of likelihood ratio test statistic $G^{2}$ for testing goodness-of-fit each model. From Table 2, we see that the S model fits these data poorly. So, we are interested in the cause that the $\mathrm{S}$ model fits poorly.

First, we consider the decomposition of the $\mathrm{S}$ model using the models which indicate the structure of symmetry (namely, Caussinus's (1965) decomposition, Theorem 2 and 3). From Table 2, the QS and MH models fit these data poorly. Thus, from Caussinus's decomposition, it is impossible to specify either the decomposed two models (QS and MH models) about the cause model that the S model fits poorly. However, using Theorem 2, we can see that the poor fit of the $\mathrm{S}$ model is caused by the influence of lack 
Table 2. Likelihood ratio chi-square values $G^{2}$ for models applied to the data in Table 1 .

\begin{tabular}{ccc}
\hline Applied models & df & $G^{2}$ \\
\hline S & 6 & $750.56^{*}$ \\
CS & 5 & $362.60^{*}$ \\
QS & 3 & $8.30^{*}$ \\
MH & 3 & $742.76^{*}$ \\
ROS & 3 & $193.14^{*}$ \\
RMS & 3 & $557.43^{*}$ \\
RMAS & 2 & $169.46^{*}$ \\
COS & 3 & 6.30 \\
CMS & 3 & $744.26^{*}$ \\
CMAS & 2 & $356.29^{*}$ \\
GS & 1 & $387.97^{*}$ \\
\hline
\end{tabular}

* means significant at the 0.05 level.

of the CMS model rather than the COS model. Thus, it is seen that in these data there is not a structure of symmetry of cell probabilities $\left\{p_{i j}\right\}$ but there is a structure of symmetry of odds $\left\{p_{i-1, j} / p_{i j}\right\}$.

Next, we consider the decomposition of the $\mathrm{S}$ model using the models which indicate the structure of asymmetry (namely, Read's (1978) decomposition). From Read's decomposition, we cannot see the cause that the S model fits poorly. Because the CS and GS models fit these data poorly. So, we are interested in considering the cause that the CS model fits poorly using Theorems 3 and 4. From Theorem 4, we can see that the poor fit of the CS model is caused by the influence of lack of the CMAS model rather than the COS model. Therefore, from Corollary 2, we can see that the poor fit of the S model is caused by the influence of lack of the CMAS and GS models rather than the COS model.

\section{Concluding Remarks}

We point out from Theorem 7 that the likelihood ratio statistic for testing goodness-of-fit of the $\mathrm{S}$ model assuming that the ROS model holds true is $G^{2}(S)-G^{2}(R O S)$ and this is equal to the likelihood ratio statistic for testing goodness-of-fit of the RMS model, i.e., $G^{2}(R M S)$. Namely, $G^{2}(R M S)$ can be used for testing goodness-of-fit of the RMS model and also for testing goodness of fit the $\mathrm{S}$ model assuming that the ROS model holds true. Theorems 8 to 12 also have similar properties.

Generally suppose that model $M_{3}$ holds if and only if both models $M_{1}$ and $M_{2}$ hold, where the number of df for $M_{3}$ equals the sum of numbers of df for $M_{1}$ and $M_{2}$. Darroch and Silvey (1963) described that (1) when the asymptotic equivalence

$$
G^{2}\left(M_{3}\right) \simeq G^{2}\left(M_{1}\right)+G^{2}\left(M_{2}\right)
$$

holds, if both $M_{1}$ and $M_{2}$ are accepted (at the $\alpha$ significance level) with high probability, then $M_{3}$ would be accepted; however, (2) when (5.1) does not hold, it is quite possible for an incompatible situation to arise where both $M_{1}$ and $M_{2}$ are accepted with high probability but $M_{3}$ is rejected with high probability (in fact, Darroch and Silvey (1963) showed such an interesting example). For Theorems 7 to 12, such an incompatible situation would not arise.

Tomizawa (1984) gave decompositions of the S model using QS and RMS (or CMS). For the analysis of data, decompositions of the S model given by this paper may be preferable to Tomizawa's (1984) decompositions, because the goodness-of-fit test statistic for the $\mathrm{S}$ model is not equal (or asymptotically equivalent) to the sum test statistics for decomposed models by Tomizawa's (1984). In fact, for the artificial data in Table 3, we see from Table 4 that the $\mathrm{S}$ model is rejected though both QS and RMS models are accepted. 
Table 3. Artificial data.

\begin{tabular}{cccccc}
\hline & $(1)$ & $(2)$ & $(3)$ & $(4)$ & Total \\
\hline$(1)$ & 300 & 99 & 53 & 56 & 508 \\
$(2)$ & 90 & 300 & 67 & 78 & 535 \\
$(3)$ & 73 & 93 & 300 & 30 & 496 \\
$(4)$ & 54 & 38 & 20 & 300 & 412 \\
\hline Total & 517 & 530 & 440 & 464 & 1951 \\
\hline
\end{tabular}

Table 4. Likelihood ratio chi-square values $G^{2}$ for models applied to the data in Table 3.

\begin{tabular}{ccc}
\hline Applied models & df & $G^{2}$ \\
\hline S & 6 & $23.99^{*}$ \\
QS & 3 & 7.18 \\
MH & 3 & $16.75^{*}$ \\
ROS & 3 & $21.08^{*}$ \\
RMS & 3 & 2.91 \\
COS & 3 & 6.28 \\
CMS & 3 & $17.72^{*}$ \\
\hline
\end{tabular}

* means significant at the 0.05 level.

Acknowledgments. The authors would like to thank the referee for their helpful comments.

\section{References}

1. Bishop, Y. M. M., Fienberg, S. E., and Holland, P. W. (1975). Discrete Multivariate Analysis: Theory and Practice. Cambridge, Massachusetts: The MIT Press.

2. Bowker, A. H. (1948). A test for symmetry in contingency tables. Journal of the American Statistical Association, $43,572-574$.

3. Caussinus, H. (1965). Contribution à l'analyse statistique des tableaux de corrélation. Annales de la Faculté des Sciences de l'Université de Toulouse, 29, 77-182.

4. Darroch, J. N. and Silvey, S. D. (1963). On testing more than one hypothesis. Annals of Mathematical Statistics, 34, 555-567.

5. Kateri, M, Gottard, A, and Tarantola, C. (2017). Generalised quasi-symmetry models for ordinal contingency tables, Australian \& New Zealand Journal of Statistics, 59, 239-253.

6. McCullagh, P. (1978). A class of parametric models for the analysis of square contingency tables with ordered categories. Biometrika, 65, 413-418.

7. Read, C. B. (1977). Partitioning chi-square in contingency tables: A teaching approach. Communications in Statistics - Theory and Methods, 6, 553-562.

8. Stuart, A. (1955). A test for homogeneity of the marginal distributions in a two-way classification. Biometrika, $42,412-416$.

9. Tahata, K. and Tomizawa, S. (2014). Symmetry and asymmetry models and decompositions of models for contingency tables. SUT Journal of Mathematics, 50, 131-165.

10. Tominaga, K. (1979). Nippon no Kaisou Kouzou (Japanese Hierarchical Structure). University of Tokyo press, Tokyo (in Japanese).

11. Tomizawa, S. (1984). Three kinds of decompositions for the conditional symmetry model in a square contingency table. Journal of the Japan Statistical Society, 14, 35-42.

12. Tomizawa, S. (1985). Decompositions for odds-symmetry models in a square contingency table with ordered categories. Journal of the Japan Statistical Society, 15, 151-159.

13. Tomizawa, S., and Tahata, K. (2007). The analysis of symmetry and asymmetry: orthogonality of decomposition of symmetry into quasi-symmetry and marginal symmetry for multi-way tables. Journal de la Société Française de Statistique, 148, 3-36. 